TP Periodica Polytechnica Chemical Engineering

60(3), pp. 210-217, 2016

DOI: $10.3311 /$ PPch.8637

Creative Commons Attribution (i)

RESEARCH ARTICLE

\section{Numerical Investigation of Mixing Time in a Torus Reactor}

\author{
Khalida Bekrentchir $^{1 *}$, Abdelkader Debab ${ }^{1}$
}

Received 05 October 2015; accepted after revision 07 January 2016

\begin{abstract}
In this work, the mixing performance in a batch torus reactor was investigated using computational fluid dynamics (CFD). To validate the numerical model, the CFD results for the mean bulk velocity and power number were compared with the experimental data reported in the literature. Next, by solving numerical dispersion of a passive tracer in unsteady state, the effects of important parameters such as the impeller type, impeller diameter, impeller blade angle and impeller rotation speed on the mixing time were explored. The analysis of numerical results allows us to suggest simple correlations for the prediction of the mixing time as a function of the total specific power consumption $P / V$, impeller diameter to reactor diameter ratio $D / d$ and impeller blade angle $\sin \varphi$.
\end{abstract}

\section{Keywords}

CFD, Torus reactor, Impeller type, Flow pattern, Mixing time, Power consumption

\footnotetext{
${ }^{1}$ Laboratoire d'Ingénierie des Procédés de l'Environnement, Université des Sciences et de la Technologie Mohamed Boudiaf, BP 1505 El Mnaouer, Oran, Algeria

${ }^{\text {*} C o r r e s p o n d i n g ~ a u t h o r, ~ e-m a i l: ~ k . b e k r e n t c h i r @ g m a i l . c o m ~}$
}

\section{Introduction}

Torus reactor has emerged as one of the most promising devices in chemical, biochemical and environmental engineering operations. It is particularly well-suited for processes that demand rapid and uniform distribution of the reaction components, e.g., polymerization [1], and multiphase systems that require high mass and heat transfer, e.g., enzymatic and photobiological reactions [2-4]. The combination of the swirl flow generated by the impeller rotation with Dean vortices that appear when an axial flow occurs in the bends efficiently mixes the reactants, produces intense dispersing effects and leads to an absence of dead volumes in the reactor $[5,6]$. Furthermore, the loop configuration involves a low-pressure drop, which reduces power consumption for a given mixing operation [7].

Sato et al. [8] were the first to study the flow pattern in the torus reactor. They developed an empirical correlation based on a simple discharge flow model to predict the mean bulk velocity as a function of the type of the impeller, blade angle, impeller diameter, impeller speed and baffle. This model indicates that the resulting flow is mainly a function of the impeller speed and the sine of the impeller blade angle. Murakami et al. [9] predicted the effect of the reactor geometry, baffles, impeller type, blade angle, impeller diameter, impeller speed and operating mode on the power consumption. They found that the power number is proportional to $(\sin \varphi)^{2}$ in the region of high mixing Reynolds number $\left(\operatorname{Re}>10^{4}\right)$; where $\varphi$ is the impeller blade angle and almost independent of the baffles and operation mode. Tanaka et al. [10] used the ratio of the circulation Reynolds number to the mixing Reynolds number to explore the effect of the reactor diameter, impeller diameter, impeller speed and fluid properties on the mean bulk velocity. They also developed a new correlation relate the power consumption in the torus reactor to the impeller speed and geometrical characteristics of the reactor and the impeller. Other researchers investigated the hydrodynamic characteristics of the torus reactor using residence time distribution (RTD) technique. Belleville et al. [11] employed the axial dispersed plug flow with complete circulation to model the batch torus reactor and proposed a new correlation for the dimensionless mixing 
time on the basis of Kolmogoroff's theory to predict the effect of the geometric characteristics of the reactor and the impeller on the mixing time in the torus reactor. They also improved the correlation proposed by Sato et al. [8] by introducing a geometrical parameter to relate the dimensions of the impeller and the torus reactor. Benkhelifa et al. [12] studied the flow pattern in batch and a continuous torus reactor. They observed that for a ratio of the mean residence time to the mean circulation time lesser than 20, the flow is accelerated by the inlet-outlet flow rate. Khalid et al. [5, 6] investigated the local flow properties of the torus reactor using residence time distribution (RTD) technique and electrochemical probes. They observed that the bend curvature affect the mains features of the flow, practically the mean velocity profiles, wall pressure and turbulent characteristics. Recently, Pruvost et al. [13] and Pramparo et al. [14] employed computational fluid dynamics (CFD) model to investigate the mixing characteristics in a square-sectioned torus reactor in batch and continuous configurations. Obtained results confirm that CFD modelling ensure an improved prediction of mixing characteristics in toroidal reactors. Such a tool was also successfully applied to evaluate the mixing time in a gas-liquid milli torus reactor operated without agitation [15].

In this study, CFD was utilized to investigate the effects of different operating conditions and design parameters on the mixing performance of a circular-sectioned torus reactor. To validate the model, the CFD results for the mean bulk velocity and power consumption were compared with the experimental data reported in the literature [10]. The validated CFD model was then used to explore the dependence of mixing time and power consumption on impeller type, impeller diameter and blade angle. To the knowledge of the authors, no study has been reported in the literature on the mixing time and power consumption calculations in the torus geometry using CFD technique although these parameters provide useful information regarding the design, modelling, optimization and scale up of stirred reactors.

\section{Numerical details}

\subsection{Geometrical details and grid consideration}

The investigated torus reactor is described in Tanaka et al. [10]. It consists of four $90^{\circ}$ bends having a constant circular cross-section with an inner tube diameter $D(=2 a)$; curvature ratio, $\gamma\left(=R_{C} / a\right)$; and a loop length $\left(L_{t}\right)$, and the reactor volume is 81 (Fig. 1a). The fluid is circulated by rotating an axial flow impeller. Tap water at $30{ }^{\circ} \mathrm{C}$ was used as the working fluid. The reactor diameter and curvature ratio are maintained constant in all simulations.

Two types of axial flow impeller are used in this study, both of which have diameters of $0.032 \mathrm{~m}$ and a blade pitch angle of $45^{\circ}$. The first impeller is classical (marine impeller) with three pitched flat blades. The second impeller is a four-blade pitchedblade turbine (PBT) (Fig. 1b). These impellers are selected because they generate drastically different flow patterns. In addition, they are frequently used in industrial flow-sensitive operations, e.g., homogenization of miscible liquids [16] or suspension of solids in a liquid [17].

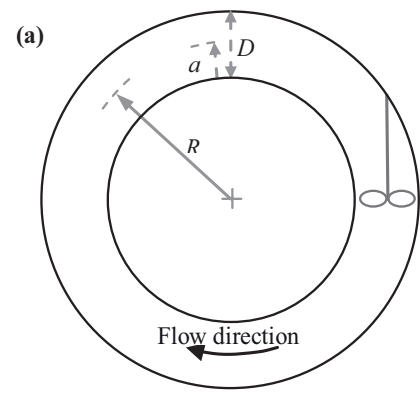

Curvature radius $\left(R_{c}\right): 0.16 \mathrm{~m}$ Inner tube radius $(a): 0.025$

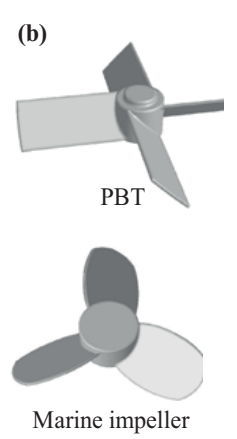

Marine impeller
Fig. 1 a) Schematic representation of the torus reactor, b) impellers investigated in the present work

The geometry model of the torus reactor and its mesh are created using GAMBIT. Because we use the MRF resolution, the reactor was divided in to non-overlapping regions, a rotating cylindrical volume that encloses the retreat impeller and an outer stationary volume that contains the remaining parts of the reactor. The interface that separates the two regions was located midway between the impeller tip and the reactor walls, which is consistent with Oshinowo et al. [18]. The outer stationary volume was also divided into two parts: an irregular part near the impeller and a regular part that corresponded to the remaining volume. A regular mesh with elementary hexahedral volumes was used in the regular part.

The grid discretization in this zone is uniform, whereas the cell density gradually increased at distances closer to the impeller. In the irregular part, hybrid meshes were retained, with an irregular zone composed of tetrahedral volumes and prisms. Additional mesh refinements near the reactor walls and impeller surfaces are completed to improve the accuracy in the high-velocity gradient zones. The MRF zone and the regularand irregular-part meshes are illustrated in Fig. 2, which clearly shows that finer meshes were formed around the impeller.

\subsection{Boundary conditions and numerical details}

The torus reactor is of closed type, and the flow result from the impeller rotation is the only driving mechanism. The only boundary condition to be specified in this case was the rotation speed of the impeller. Indeed, with the MRF resolution, the impeller remains stationary, whereas the rotating frame has an angular velocity equal to the rotation speed of the impeller. The reactor walls and the impeller surfaces are treated as nonslip boundaries with standard wall functions. Next, the commercial CFD software package FLUENT 6.3 was used to solve the continuity and momentum equations. Based on the mesh created using GAMBIT, Fluent uses a finite-volume method to discretize the previously described governing equations over 
each cell and the discrete equations are solved using a numerical tool with the boundary conditions. In all simulations, the segregated solver is used to iterate the nonlinear partial differential equations since this solver is better adapted to the grids used in our work. To calculate the pressure at the nodes the body-force-weighted scheme is used, which works well when inertial forces are not negligible. The SIMPLEC algorithm is used for pressure-velocity coupling in order to obtain the pressure in the continuity equation. For the momentum equation, turbulent kinetic energy and energy dissipation, the second order quick scheme is used because it is recommended in the presence of rotating flows. The solutions are considered converged when repeated iterations do not change the power consumption and the dimensionless velocity residuals remain constant above $10^{-6}$. To facilitate the solution convergence for a fully turbulent regime, the rotational velocity of the impeller was increased in two or three successive steps depending on the convergence difficulties. Intermediate converged solutions were used to initialize the hydrodynamic values for the next higher rotation velocity.

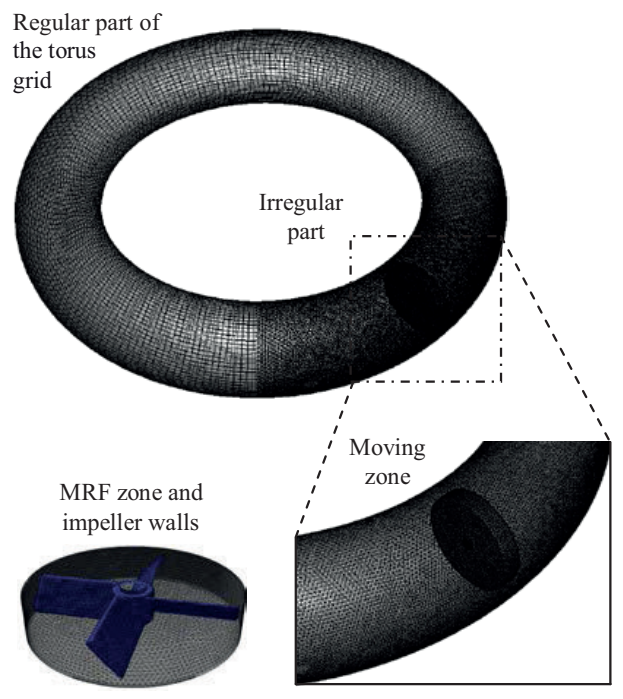

Fig. 2 Mesh topology

To simulate the mixing times after the convergence of the flow field, the unsteady state transport of an inert tracer superimposed on the calculated flow field was monitored until complete homogenization was achieved. The unsteady distribution of the tracer was determined by solving the species transport equation, based on the assumption that the tracer is distributed by convection and diffusion:

$$
\frac{\partial C}{\partial t}+U_{i} \frac{\partial C}{\partial x_{i}}=\frac{\partial}{\partial x_{i}}\left[\left(D_{j}+\frac{\mu_{t}}{S c_{t}}\right) \frac{\partial C}{\partial x_{i}}\right]
$$

where $C$ is the concentration of the passive tracer, $D_{j}$ is the usual mass diffusion coefficient in the laminar regime, and $U_{i}$ is the resolved mean velocity component $i$. The local values of the turbulent viscosity $\mu_{t}$ are obtained from the turbulence model, and only the turbulent Schmidt number values must be defined to calculate the turbulent mass diffusivity $D_{j t}\left(S c_{t}=\mu_{t} / \rho D_{j t}\right)$. Different $S c_{t}$ values from 0 to 1 have been used in this study, depending on the flow regime [19].

The mixing simulation was started by instantaneously adding the tracer at the center of the first bend outlet. Because a passive tracer is used, the mass transport equation does not affect the flow field; thus, only the mass transport equation is solved using the converged-flow-field results. To determine the evolution of the average tracer concentration with respect to time, an unsteady resolution was performed. The second-order implicit scheme was used for time discretization. For each simulated case, different resolutions were performed to verifier that the time step was sufficiently small to not affect the dispersion calculation. The time evolution of the tracer concentration was recorded at the same position as the punctual tracer injection: at the first bend outlet. Before starting the final CFD simulations, several exploratory simulations were performed using the MRF approach to conduct grid independence tests. The grid independence was verified by demonstrating that additional grid lines near the impeller surfaces and bend curvature did not change the calculated mean bulk velocity and power number by more than $5 \%$. The mean bulk velocity was computed using $U_{0}=L_{t} / t_{c}$, where $L_{t}$ is the loop length and $t_{c}$ is the mean circulation time, the mean time necessary for a fluid element to make a complete circulation in the torus reactor. For example, for a marine impeller at $R e_{m}=30000$, the original 3D mesh had 701496 cells. To verify the grid independency, their number increased to 741074 , then to 773004 cells. This increase changed the mean bulk velocity and power number by more than $5 \%$. When the number of cells was further increased to 792484 by adapting the grid three times, the mean bulk velocity and power number did not changed by more than $5 \%$. The data from Table 1 confirm that this grid satisfies the established criterion for solution independence. Therefore, grid 3 (792484 cells) was thus retained for the numerical study. The same approach was used to determine the optimum number of cells for the $45^{\circ}$ - pitched-blade turbine. A coarsened mesh having 808115 cells was retained for further investigation. It must be noticed that results for this impeller are not presented in Table 1.

Table 1 Grid independence

\begin{tabular}{|c|c|c|c|c|}
\hline $\begin{array}{l}\text { Grid } \\
\text { adaption }\end{array}$ & $\begin{array}{l}\text { Estimated no. of } \\
\text { grid cells within } \\
\text { (normal to) } 2 \\
\text { mm of impeller } \\
\text { surface }\end{array}$ & $\begin{array}{l}\text { Total } \\
\text { number of } \\
\text { cells }\end{array}$ & $\begin{array}{l}\text { CFD: } \\
\text { Mean bulk } \\
\text { velocity } \\
(\mathrm{m} / \mathrm{s})\end{array}$ & $\begin{array}{l}\text { CFD: } \\
\text { power no. }\end{array}$ \\
\hline 1 & 4 & 741074 & 0.565 & 0.94 \\
\hline 2 & 8 & 773004 & 0.524 & 0.89 \\
\hline 3 & 16 & 792484 & 0.512 & 0.87 \\
\hline
\end{tabular}




\section{Results and discussion}

\subsection{Characterization of the flow in the torus reactor}

To validate the CFD model developed in this study, the CFD results for the mean bulk velocity and also power number were compared with the experimental data reported in the literature. As depicted in Fig. 3, results for the mean bulk velocity $U_{0}$ are compared to the correlation proposed by Tanaka et al. [10]. This correlation characterizes the dependence of the mixing conditions to the flowing characteristics in the torus reactor as follows:

$$
\frac{R e}{R e_{m}}=\left(\frac{C_{1}}{R e_{m}^{C_{2}}}+C_{3}\right)^{-1}
$$

where $R e$ is the circulation Reynolds number based on the mean bulk velocity and reactor diameter, $R e_{m}$ is the mixing Reynolds number, $c_{1}, c_{2}$ and $c_{3}$ are constant values depending on the type of impeller and on the geometrical characteristics of the reactor (Table 2). The validation was performed for blade angle and impeller diameter to reactor diameter ratio of $45^{\circ}$ and 0.64 , respectively.

Table 2 Values of the coefficients in Eq. (2) [10]

\begin{tabular}{llll}
\hline & $\mathrm{C}_{1}$ & $\mathrm{C}_{2}$ & $\mathrm{C}_{3}$ \\
\hline Marine impeller & $2.310^{2}$ & 0.7 & 1 \\
PBT & $2.710^{2}$ & 0.7 & 1 \\
\hline
\end{tabular}

Results in Fig. 3 are in good agreement with this correlation, confirming the choice of the MRF method for the impeller modelling. If results obtained between the two impellers are compared, a more efficient flow circulation is obtained with the marine impeller. This is certainly explained by the special design of marine impeller, which is theoretically better shaped to promote an efficient axial flow circulation. Finally, it must be noticed that the ratio of the circulation Reynolds number to the mixing Reynolds number is inversely proportional to the Reynolds number for low mixing Reynolds numbers and becomes nearly independent of the impeller rotation speed for $R e_{m}$ greater than $1.510^{4}$. Thus, depending on the $R e_{m}$ values, the hydrodynamic in the torus reactor can be characterized by three different flow regimes: a laminar regime for mixing Reynolds numbers below $10^{4}$, a turbulent regime for $R e_{m}>1.510^{4}$, and a transient regime from laminar behavior to turbulent behavior for the mixing Reynolds number range of $10^{4}-1.510^{4}$, where this ratio depends on the Reynolds number.

CFD results for the power number were also compared with the experimental data of Tanaka et al. [10] at $R e_{m}>1 \times 10^{4}$. It must be mentioned that the power consumed by each impeller was calculated using $N_{P}=2 \pi M / \rho N^{2} d^{5}$, where $M, N$ and $d$ are the moment vector about the center of the impeller, impeller speed and impeller diameter, respectively. As shown in Table 3, these results show good agreement between values obtained numerically and Tanaka et al. [10] experimental data.

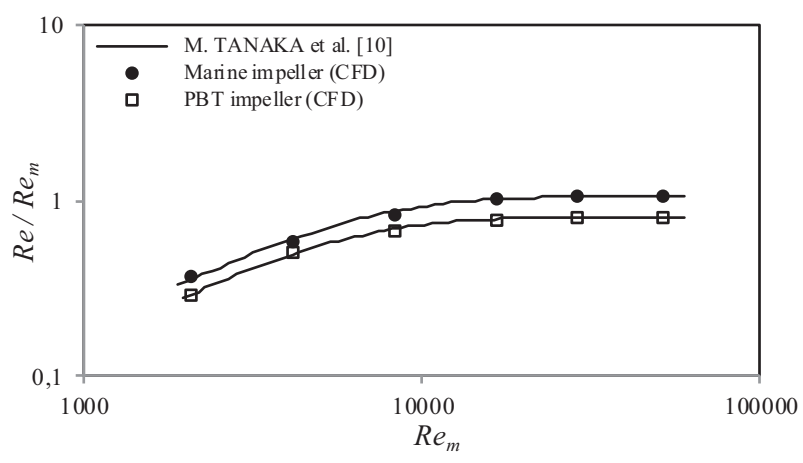

Fig. 3 Ratio of the circulation Reynolds number to the mixing Reynolds number as a function of the mixing Reynolds number: comparison with the correlation of Tanaka et al. [10]

Table 3 Computed and experimental power number reported by Tanaka et al. [10]

\begin{tabular}{lllll}
\hline & \multicolumn{4}{c}{ Power number } \\
\cline { 3 - 5 } Impeller type & $R e_{m}$ & Tanaka et al. [9] & CFD & $\begin{array}{l}\text { Relative } \\
\text { error (\%) }\end{array}$ \\
\hline Marine impeller & $R e_{m}>1 \times 10^{4}$ & 0.85 & 0.87 & 2.35 \\
PBT & $R e_{m}>1 \times 10^{4}$ & 1.15 & 1.18 & 2.6 \\
\hline
\end{tabular}

\subsection{Investigation of mixing in the torus reactor}

In this part, the numerical tool is used to investigate the effect of the impeller type, impeller diameter and blade angle on the mixing performance of the torus reactor. To evaluate the efficiency of the different mixing systems, the original Nienow's model [16] is extended to the case of the torus reactor geometry. This model related the evolution of the mixing time to the specific power consumption by the flowing equation:

$$
t_{m}=b\left(\frac{P}{V}\right)^{-1 / 3}
$$

where $t_{m}$ is the mixing time, $P / V$ is specific power consumption and $b$ is a constant value depending on the type of impeller and on the geometrical characteristics of the reactor. The proportionality constant $b$ is determined by fitting the experimental data of mixing time with this equation. Figure 4 shows the evolution of the mixing and circulation time as a function of the specific power consumption for the marine impeller and pitched-blade turbine.

As shown by values of mixing time, the marine impeller appears to gives longer mixing time for a given specific power input. The pitched-blade turbine allows thus an efficient mixing compared to the marine impeller. This is explained by the intense turbulence involved by the pitched-blade turbine, which gives rise to an enhancement of the mixing efficiency in the torus reactor. Based on Eq. (3), the evolution of the mixing is correlated in terms of specific power consumption and the quantitative correlations for both impellers are given in Fig. 4. The comparison of $b$ values obtained in the present study with classical results achieved in stirred tanks shows that the mixing 
time in the torus reactor remains largely higher than in stirred tanks equipped with pitched-blade turbine, where $b$ values are in the range of 80-100 [20]. This confirms the results obtained by Boesinger et al. [21], where they compared the performance of the torus and the stirred batch reactor on the basis of equal power consumption. If results for circulation time are considered, despite the intense pumping effect of the marine impeller, an almost similar circulation time is observed for both impellers. This is certainly explained by the resulting surface of pumping (number of blades), which is higher in the case of the pitched-blade turbine, and thus compensate the difference in axial pumping efficiency with the marine impeller.

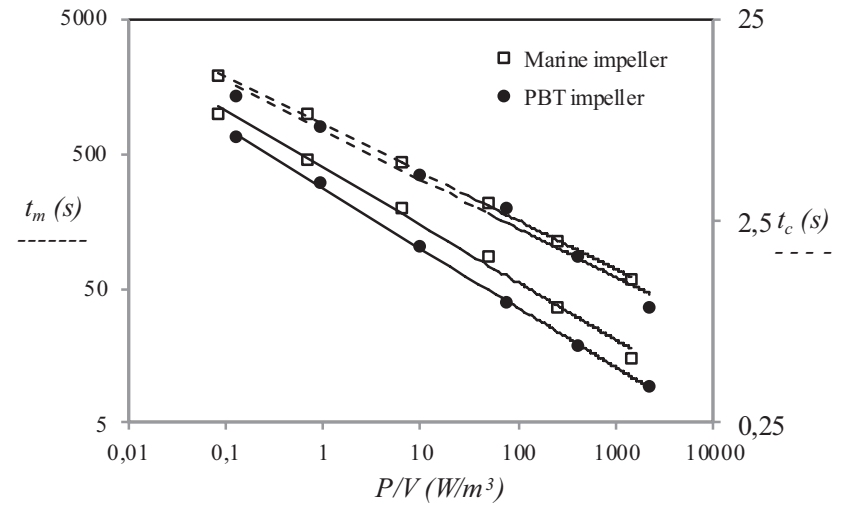

Fig. 4 Predicted mixing and circulation times as a function of the specific power consumption

The impeller diameter plays an important role in the overall flow pattern, which affects directly the mixing time and power consumption. In this work, the effect of impeller diameter on the mixing time was studied using the $45^{\circ}$ - pitched-blade turbine. The values of impeller diameter to reactor diameter ratios used were respectively $0.33,0.5,0.64$ and 0.75 . To determine the optimum value of the impeller diameter to reactor diameter ratio $d / D$, values of mixing time are presented in Fig. 5 as function of the impeller diameter to reactor diameter ratio $d / D$.

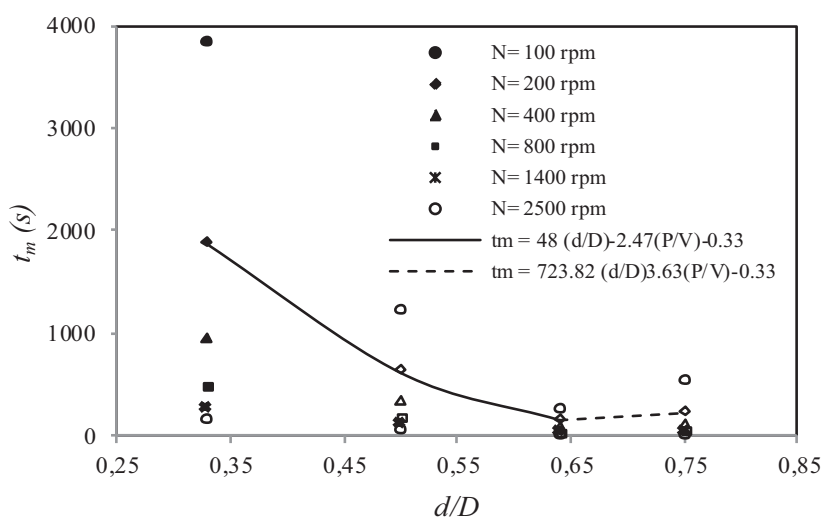

Fig. 5 Effect of impeller diameter to reactor diameter ratio on the mixing time $\left(\varphi=45^{\circ}\right)$
These results show that, the mixing time achieved by the pitched-blade turbine with $d / D$ ratio of 0.64 is less than that achieved for the pitched-blade turbine with higher $d / D$ ratio, which is found to give shorter mixing time compared to the impellers with $d / D$ ratio of 0.5 and 0.33 . The decrease in the mixing efficiency for large impeller diameter can be explained by the change in the flow involved downstream the pitchedblade turbine due to the impeller diameter to reactor diameter ratio, which is found to be closer to radial flow if $d / D$ ratio is increased above 0.55 [22]. To verify this, the flow structures generated by $d / D=0.5$ and 0.75 impellers ware represented in Fig. 6 by plotting velocity-fields in the impeller region for $R e_{m}=22721$.
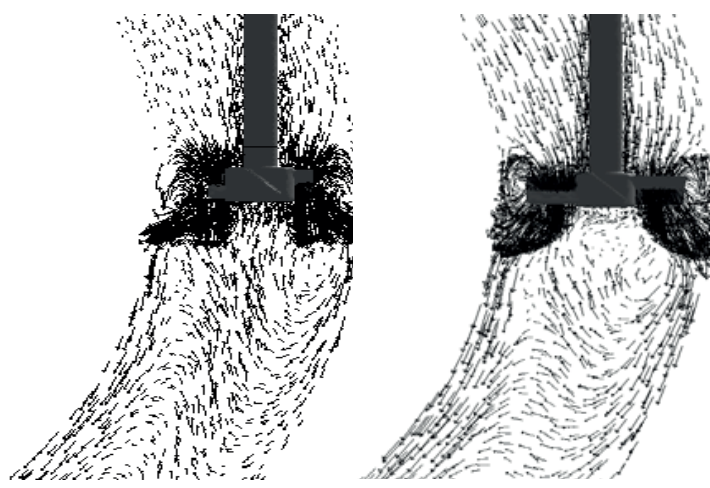

Fig. 6 Flow structure in the impeller region for $R e_{m}=22721$

(left: $d / D=0.5$; right $d / D=0.75$ )

The difference in flow structure is clearly shown. For the smaller impeller $(d / D=0.5)$ the flow downstream the impeller is discharged in both axial and radial directions and a small secondary circulation loop is formed in the outer part of the bend, whereas, for the larger one $(d / D=0.75)$ the deflection of the discharge flow angle from the axial direction toward the radial direction increased the size and the intensity of the secondary circulation loop, which results in a poor circulation in the torus reactor, and thus higher values of mixing time. These flow patterns agree well with the experimental results obtained by Mao et al. [23], Kresta et al. [24] and Tsui et al. [25]. Finally, it must be noticed that the high values of mixing time obtained for the small impeller diameter to reactor diameter ratio $(d / D=0.33$ and $d / D=0.5)$, particularly for low impeller speed, are explained by the poor flow circulation in those conditions due to the trapping of fluid particles in the swirl flow involved downstream of the pitched-blade turbine.

In order to obtain the quantitative relationship between the mixing time $t_{m}$ and the impeller diameter to reactor diameter ratio, the mixing time $t_{m}$ could be correlated by Eq. (4):

$$
t_{m}=\alpha\left(\frac{d}{D}\right)^{\beta}\left(\frac{P}{V}\right)^{\gamma}
$$


Compared with the numerical results (Fig. 5), two correlations are obtained:

$$
\begin{array}{ll}
t_{m}=48\left(\frac{d}{D}\right)^{-2.47}\left(\frac{P}{V}\right)^{-0.33} & \frac{d}{D} \leq 0.65 \\
t_{m}=723.82\left(\frac{d}{D}\right)^{3.63}\left(\frac{P}{V}\right)^{-0.33} & \frac{d}{D} \geq 0.65
\end{array}
$$

It can be seen that the absolute exponent of $d / D$ is larger than that of the specific power consumption which means that the effect of the $d / D$ on mixing time is greater than that of the specific power consumption. Equation (6) also shows that, the power exponent of $d / D$ is positive, reflecting the increase of mixing time for the larger impeller diameter $(d / D>0.64)$.

The effect of the impeller blade angle is investigated next using the pitched-blade turbine with $d / D$ ratio of 0.64 . The blade pitch angles considered are $\varphi=30^{\circ}, \varphi=45^{\circ}$ and $\varphi=60^{\circ}$. Values of mixing time obtained for different conditions are presented as function of $\sin \varphi$ in Fig. 7. This result shows that, the mixing time is proportional to the square of $\sin \varphi$ and the proportionality constant depends on the impeller rotation speeds.

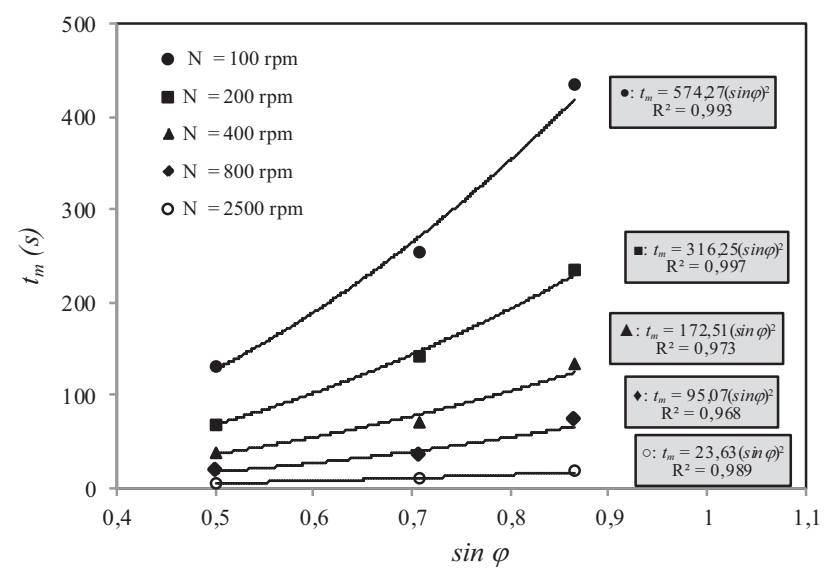

Fig. 7 Effect of impeller angle on the mixing time $(d / D=0.64)$

To show the effect of the specific power consumption, impeller diameter to reactor diameter ratio and impeller blade angle on the mixing time, Eq. (4) is extended to

$$
t_{m}=\alpha\left(\frac{d}{D}\right)^{\beta}\left(\frac{P}{V}\right)^{\gamma}(\sin \varphi)^{\delta}
$$

Based on numerical results of this study, two correlations of mixing time are given by

$$
\begin{aligned}
& t_{m}=75.86\left(\frac{d}{D}\right)^{-2.47}\left(\frac{P}{V}\right)^{-0.33}(\sin \varphi)^{1.55} \quad \frac{d}{D} \leq 0.65 \\
& t_{m}=1144.43\left(\frac{d}{D}\right)^{3.63}\left(\frac{P}{V}\right)^{-0.33}(\sin \varphi)^{1.55} \quad \frac{d}{D} \geq 0.65
\end{aligned}
$$

Figure 8 shows the comparison between $t_{m}$ obtained from numerical investigation and those predicted by the empirical correlation. All the predicted data points lay within $\pm 10 \%$ deviation lines of the numerical results. The correlation is applicable in the $P / V$ range of $0.05-4000 \mathrm{~W} / \mathrm{m}^{3}, d / D$ range of 0.33 0.75 and $\sin \varphi$ range of $0.50-0.87$. Further investigations are recommended to minimize the error for the application of correlation in the wide range of $P / V$ and $d / D$.

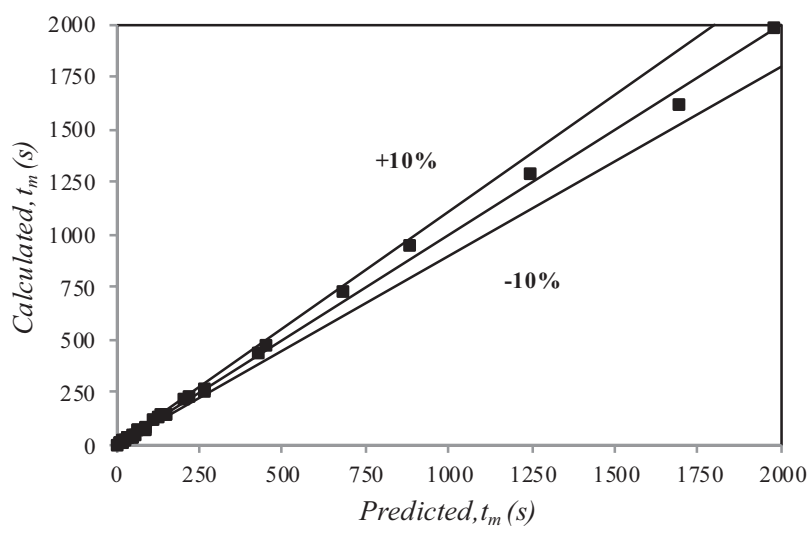

Fig. 8 Comparison of the correlated mixing time by Eq. (8) and Eq. (9) with numerical data

\subsection{Investigation of power consumption in the torus reactor}

The power consumption is another crucial characteristic of stirred reactors. This global parameter is particularly useful when comparing the mixing in stirred reactor equipped with different types of impellers. The power consumption $P$ is calculated as the product of torque derived from the pressure and tangential stress distribution on the moving surfaces (impeller blades and shaft), with the angular velocity:

$$
P=2 \pi N \int_{A} r \times(\tau d A)
$$

where $A$ is the overall impeller and shaft surface area, $r$ the position vector, and $\tau$ the stress tensor. To investigate the dependence of the power consumption on the impeller speed and the reactor geometry, the predicted values of the power consumption are shown as a function of the impeller speed in Fig. 9. These results show that, the power consumption increases with the increase of the impeller speed and the impeller diameter to reactor diameter ratio, and is proportional to $N^{3}(d / D)^{4.5}$.

As reported before, Murakami et al. [9] have found that the dependence of the power number on the mixing Reynolds number was similar to that obtained in the conventional stirred tank reactor [26], and the power number was proportional to $(\sin \varphi)^{2}$. Similar observations were achieved by Tanaka et al. [10], while the exponent of $(\sin \varphi)$ was 1.5 . Thus, as in stirred tanks studies, the power consumption in the torus reactor can be expressed as follows:

$$
P=K D^{\varepsilon} N^{\vartheta}\left(\frac{d}{D}\right)^{\sigma}(\sin \varphi)^{\omega}
$$




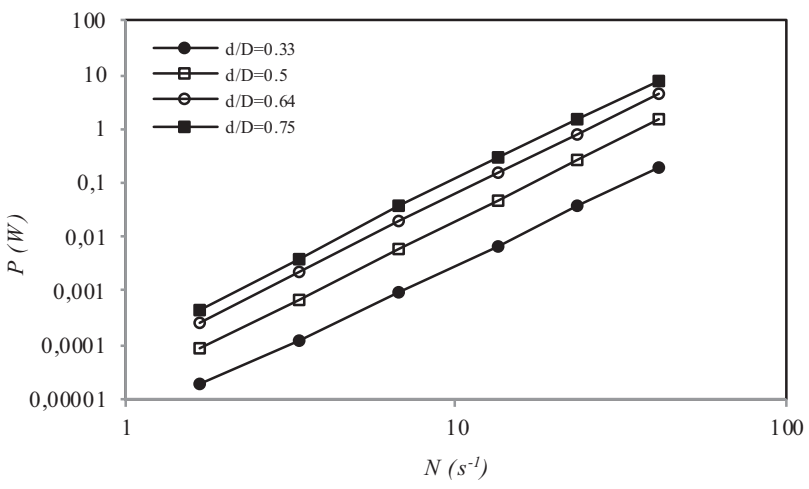

Fig. 9 Effect of impeller speed and impeller diameter to reactor diameter ratio on the power consumption

Based on the numerical results, the power consumption for the pitched-blade turbine is given by:

$$
P=6.998 D^{3} N^{3}\left(\frac{d}{D}\right)^{4.46}(\sin \varphi)^{2}, R^{2}=0.99
$$

Figure 10 shows the comparison between the predicted power consumption and the correlation. The reasonable agreement between Eq. (12) and the numerical data could be got with deviation within $\pm 5 \%$ in the range of $1.67 \leq N \leq 41.67 \mathrm{~s}^{-1}$, $0.33 \leq d / D \leq 0.75$ and $0.50 \leq \sin \varphi \leq 0.87$.

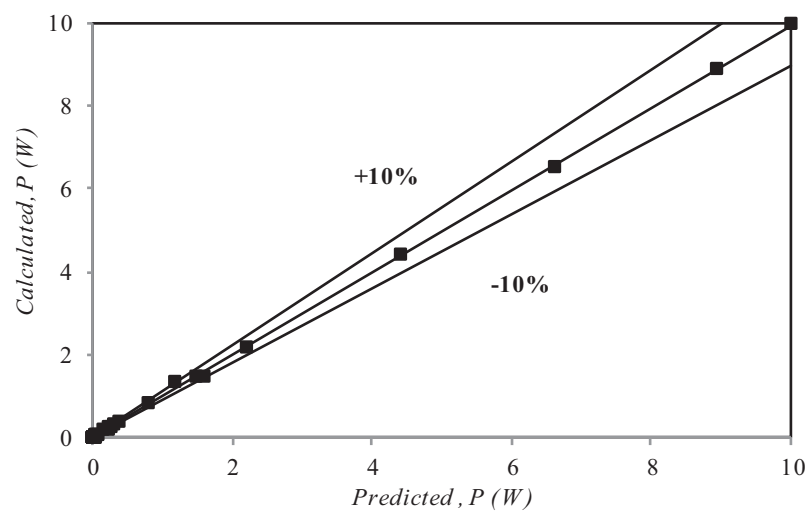

Fig. 10 Comparison of the correlated power consumption by Eq. (12) with numerical data

\section{Conclusions}

In this study, the mixing performance of the torus reactor was investigated using the Computational fluid dynamics (CFD). To explore the effects of the impeller speed, impeller type, impeller diameter and impeller blade angle on the mixing time, an unsteady resolution of a passive tracer was performed in various configurations. The following conclusions could be made,

1) All of the investigations show that the $30^{\circ}$ - pitched-blade turbine with $d / D$ ratio of 0.64 achieves a more efficient mixing for a given power consumption.

2) Predicted values of mixing time vary reversely with the cube root of the specific power consumption and are

proportional to $\sin \varphi^{1.55}$. The comparison of proportionality constants obtained in the present study with classical results achieved in stirred tanks shows that the torus reactor seems a little less efficient than stirred tanks equipped with turbine.

3) As the ratio of impeller diameter to reactor diameter $d / D$ increases from 0.33 to 0.65 , the mixing time decreases, whereas, further increases in $d / D$ increase the mixing time. Satisfactory predictions of $t_{m}$ could be obtained as

$$
\begin{array}{lll}
t_{m}=75.86\left(\frac{d}{D}\right)^{-2.47}\left(\frac{P}{V}\right)^{-0.33}(\sin \varphi)^{1.55} & \frac{d}{D} \leq 0.65 \\
t_{m}=1144.43\left(\frac{d}{D}\right)^{3.63}\left(\frac{P}{V}\right)^{-0.33}(\sin \varphi)^{1.55} & \frac{d}{D} \geq 0.65
\end{array}
$$

with $0.05 \leq P / V \leq 4000 \mathrm{~W} / \mathrm{m}^{3}, 0.33 \leq d / D \leq 0.75$ and $0.50 \leq \sin \varphi \leq 0.87$.

4) The power consumption for the pitched-blade turbine could be correlated to the impeller speed and the geometrical characteristics of the reactor and the impeller by the following relationship:

$$
P=6.998 D^{3} N^{3}\left(\frac{d}{D}\right)^{4.46}(\sin \varphi)^{2}
$$

With $1.67 \leq N \leq 41.67 \mathrm{~s}^{-1}, 0.33 \leq d / D \leq 0.75$ and $0.50 \leq$ $\sin \varphi \leq 0.87$.

The predicted $t_{m}$ and $P$ where found to be within $\pm 10 \%$ range of the numerical results. These results provide useful information for the design and optimization of the torus reactor geometry.

\section{Nomenclature}

$a$

$C_{j}$

$C_{\infty}$

d

D

$D_{j}$

$D_{j t}$

$L_{t}$

N

$N_{P}$

$P$

$r$

$R_{c}$

$R e$

$R e_{m}$

$S_{c t}$

$t_{c}$

$t_{m}$

$U_{0}$
Inner pipe radius[m]

Passive tracer concentration $\left[\mathrm{mol} \mathrm{m}^{-3}\right.$ ]

Homogeneous tracer concentration $\left[\mathrm{mol} \mathrm{m}^{-3}\right]$ Impeller diameter [m]

Pipe diameter [m]

Laminar mass diffusivity $\left[\mathrm{m}^{2} \mathrm{~s}^{-1}\right]$

Turbulent mass diffusivity $\left[\mathrm{m}^{2} \mathrm{~s}^{-1}\right]$

Length of the torus reactor [m]

Rotation velocity of the impeller [rpm]

Power number

Power input by the impeller [W]

Position vector [m]

Bend curvature radius [m]

Circulation Reynolds number $\left(=\rho D U_{0} / \mu\right)$

Mixing Reynolds number $\left(=\rho N D^{2} / \mu\right)$

Turbulent Schmidt number

Mean circulation time [s]

Mixing time [s]

Mean bulk velocity $\left[\mathrm{m} \mathrm{s}^{-1}\right]$ 


\section{Greek letters}

\section{$\varphi$}

$\mu$

$\mu_{t}$

$\rho$

\section{References}

[1] Hosogai, K., Tanaka, M. "Study of suspension polymerisation of styrene with a circular loop reactor." Polymer Engineering and Science. 32(6), pp. 431-437. 1992. DOI: 10.1002/pen.760320608

[2] Nouri, L., Legrand, J., Popineau, Y., Belleville, P. "Enzymatic hydrolysis of wheat proteins Part 2: Comparison of performance of batch-stirred and torus reactors." Chemical Engineering Journal. 65(3), pp. 195-199. 1997. DOI: $10.1016 / \mathrm{s} 1385-8947(97) 00003-\mathrm{x}$

[3] Legrand, J., Guéguen, J., Berot, S., Popineau, Y., Nouri, L. "Acetylation of pea isolate in a torus microreactor." Biotechnology and Bioengineering. 53(4), pp. 409-414. 1997.

DOI: 10.1002/(sici)1097-0290(19970220)53:4<409::aid-bit9>3.0.co;2-r

[4] Ji, C. F., Legrand, J., Pruvost, J., Chen, Z. A., Zhang, W. "Characterization of hydrogen production by Platymonas Subcordiformis in torus photobioreactor." International Journal of Hydrogen Energy. 35(13), pp. 7200-7205. 2010. DOI: 10.1016/j.ijhydene.2010.02.085

[5] Khalid, A., Legrand, J., Rosant, J. M. "Turbulent flow induced by an impeller in a closed toroidal loop." Journal of Fluids Engineering. 118(4), pp. 677-684. 1996. DOI: 10.1115/1.2835495

[6] Khalid, A., Legrand, J. "Energy dissipation distribution and mixing in a torus reactor." Chemical Engineering Communication. 185(1), pp. 141164. 2001. DOI: $10.1080 / 00986440108912860$

[7] Legrand, J., Belleville, P. "Flow characteristics and transport phenomena in toroidal loop reactors." Chemical Engineering and Technology. 25(6), pp. 667-670. 2002.

DOI: 10.1002/1521-4125(200206)25:6<667::aid-ceat667>3.0.co;2-1

[8] Sato, Y., Murakami, Y., Hirose, T., Hashigushi, Y., Ono, S., Ichikawa, M. "Flow pattern, circulation velocity and pressure loss in loop reactor." Journal of Chemical Engineering of Japan. 12(6), pp. 448-453. 1979. DOI: $10.1252 /$ jcej. 12.448

[9] Murakami, Y., Hirose, T., Ono, S., Eitoku, H., Nishijima, T. "Power consumption and pumping characteristics a loop reactor." Industrial and Engineering Chemistry Process Design and Development. 21(2), pp. 273276. 1982. DOI: $10.1021 / \mathrm{i} 200017 \mathrm{a} 010$

[10] Tanaka, M., Sendai, T., Hossogai, K. "Flowing characteristics in a circular loop reactor." Chemical Engineering Research and Design. 67(4), pp. 423-427. 1989.

[11] Belleville, P., Nouri, L., Legrand, J. "Mixing characteristics in the torus reactor." Chemical Engineering and Technology. 15(4), pp. 282-289. 1992. DOI: $10.1002 /$ ceat. 270150412

[12] Benkhelifa, H., Legrand, J., Legentilhomme, P., Montillet, A. "Study of the hydrodynamic behaviour of the batch and continuous torus reactors in laminar and turbulent flow regimes by means of tracer methods." Chemical Engineering Science. 55(10), pp. 1871-1882. 2000.

DOI: 10.1016/s0009-2509(99)00447-9
[13] Pruvost, J., Pottier, L., Legrand, J. "Numerical investigation of hydrodynamic and mixing conditions in a torus photobioreactor." Chemical Engineering Science. 61(14), pp. 4476-4489. 2006.

DOI: $10.1016 /$ j.ces.2006.02.027

[14] Pramparo, L., Pruvost, J., Stüber, F., Font, J., Fortuny, A., Fabregat, A., Legentilhomme, P., Legrand, J., Bengoa, C. "Mixing and hydrodynamics investigation using CFD in a square-sectioned torus reactor in batch and continuous regimes." Chemical Engineering Journal. 137(2), pp. 386395. 2008. DOI: 10.1016/j.cej.2007.05.013

[15] Rihania, R., Guerrib, O., Legrand, J. "Three dimensional CFD simulations of gas-liquid flow in milli torus reactor without agitation." Chemical Engineering and Processing: Process Intensification. 50(4), pp. 369376. 2011. DOI: 10.1016/j.cep.2011.02.014

[16] Nienow, A. W. "On impeller circulation and mixing effectiveness in the turbulent flow regime." Chemical Engineering Science. 52(15), pp. 2557-2565. 1997. DOI: 10.1016/s0009-2509(97)00072-9

[17] Wu, J., Zhu, Z., Pullum, L. "Impeller geometry effect on velocity and solids suspension." Chemical Engineering Research and Design. 79(8), pp. 989-997. 2001. DOI: 10.1205/02638760152721857

[18] Oshinowo, L., Jaworski, Z., Dyster, K. N., Marshall, E., Nienow, A. W. "Predicting the tangential velocity field in stirred tanks using the Multiple Reference Frames (MRF) model with validation by LDA measurements." Proceedings of $10^{\text {th }}$ European Conference on Mixing. pp. 281288. 2000. DOI: 10.1016/b978-044450476-0/50036-4

[19] Patwardhan, A. W., Joshi, J. B. "Relation between flow pattern and blending in stirred tanks." India Industrial and Engineering Chemistry Research. 38(8), pp. 3131-3143. 1999. DOI: 10.1021/ie980772s

[20] Norwood, K. W., Metzner, A. B. "Flow patterns and mixing rates in agitated vessels." American Institute of Chemical Engineers Journal. 6(3), pp. 432-437. 1960. DOI: 10.1002/aic.690060317

[21] Boesinger, C., Le Guer, Y., Legentilhomme, P. "Etude expérimentale du mélange réactif au sein d'un réacteur torique ondulé (RTO)." (Experimental study of the reactive mixture in a wavy torus reactor.) The Canadian Journal of Chemical Engineering. 81(6), pp. 1-11. 2003. (in French). DOI: 10.1002/cjce.5450810606

[22] Paul, E. L., Atiemo-Obeng, V. A., Kresta, S. M. "Handbook of Industrial Mixing Science and Practice." John Wiley and Sons, Inc., Hoboken, New Jersey, USA, 2004.

[23] Mao, D.-M., Feng, L.-F., Wang, K., Li, Y.-L. "The Mean Flow Field Generated by a Pitched Blade Turbine: Changes in the Circulation Pattern due to Impeller Geometry." The Canadian Journal of Chemical Engineering. 75(2), pp. 307-316. 1997. DOI: 10.1002/cjce.5450750205

[24] Kresta, S. M., Wood, P. E. "The Mean Flow Field Produced by a $45^{\circ}$ Pitched Blade Turbine: Change in the Circulation Pattern due to Off Bottom Clearance." The Canadian Journal of Chemical Engineering. 71(1), pp. 42-53. 1993. DOI: 10.1002/cjce.5450710107

[25] Tsui, Y.-Y., Chou, J.-R., Hu, Y.-C. "Blade Angle Effects on the Flow in a Tank Agitated by the Pitched-Blade Turbine." Journal of Fluids Engineering. 128(4), pp. 774-782. 2006. DOI: 10.1115/1.2201636 